\title{
Detección y diagnóstico de fallas en sistemas eléctricos de potencia (SEP) combinando lógica difusa, métricas y una red neuronal probabilística
}

\author{
César Octavio Hernández Morales, Juan Pablo Nieto González, \\ Elías Gabriel Carrum Siller \\ Corporación Mexicana de Investigación en Materiales S.A. de C.V. (COMIMSA), \\ Saltillo, Coahuila, México \\ cesarhdz@comimsa.com,juan.nieto@comimsa.com, eliascarrum@comimsa.com
}

\begin{abstract}
Resumen. En el presente trabajo se propone un sistema para la supervisión de una red eléctrica con cambios de carga dinámicos propuesta por la IEEE. El sistema está compuesto por dos etapas. La etapa de detección utiliza un sistema de lógica difusa y la etapa de diagnóstico hace uso de las distancias Euclidianas entre líneas con el fin de generar un patrón dentro de los elementos del sistema, el cual será clasificado por una red neuronal probabilística para determinar el tipo de falla en el sistema. La combinación de estas técnicas inteligentes es para generar un sistema más robusto y seguro. Esta metodología logra la detección e identificación de fallas simétricas y asimétricas.
\end{abstract}

Palabras clave: Detección de fallas, diagnóstico de fallas, lógica difusa, métricas, red neuronal probabilística.

\section{Introducción}

La detección y diagnóstico de fallas tiene un papel muy importante en muchas áreas industriales en las que interviene la ingeniería. Esto debido a los complejos sistemas con los que hoy en día realizan sus procesos las industrias. Dicha complejidad se presenta por el gran número de variables que intervienen en los procesos industriales y que se deben de tomar en cuenta al momento de su monitoreo. Por lo tanto es una tarea retadora y difícil, el poder identificar las variables que se encuentran fuera de las condiciones normales de operación dentro de un proceso que está siendo monitoreado. Una alternativa para el monitoreo de un numero grande de variables es el uso de técnicas de inteligencia artificial. En el presente artículo son utilizadas para hacer una detección y diagnóstico más eficiente. El objetivo del presente trabajo es generar un sistema capaz de detectar y diagnosticar las fallas en sistemas complejos basado en los datos históricos del proceso. La propuesta se aplica al monitoreo de un sistema eléctrico de potencia (SEP) propuesto por la IEEE que considera cambios de carga dinámicos. La presente metodología es una sucesión de un trabajo realizado por $[1,2]$. La metodología propuesta está compuesta por dos 
pasos. El primer paso utiliza un sistema de lógica difusa para hacer la detección de las fallas y el segundo paso da el diagnóstico final al utilizar la distancia Euclidiana entre los voltajes de las líneas para cada nodo al generar un patrón para el diagnóstico. Este patrón será clasificado en los diferentes tipos de fallas por una red neuronal probabilística para obtener un sistema robusto y seguro. En la etapa de diagnóstico se realiza una comparación de la red neuronal contra la distancia Euclidiana y la de Mahalanobis para identificar la mejor técnica para realizar el diagnóstico. La organización del trabajo es de la siguiente manera. La sección 2 hace referencia a la temática del problema y se citan algunas referencias en las cuales se han utilizado las técnicas de computación suave en aplicaciones de detección y diagnóstico de fallas, en la sección 3 se presentan las herramientas matemáticas que se emplean en la presente metodología, en la sección 4 se describe el caso de estudio, la metodología se desarrolla y se presenta la evaluación de las técnicas utilizadas en la sección 5, y en la sección 6 se dan las conclusiones.

\section{Estado del arte}

Desde los inicios de la utilización de las máquinas, la necesidad de conocer si se encuentra trabajando adecuadamente ha sido una tarea habitual para los ingenieros que controlan los procesos, dada esta necesidad se tiene el deseo de detectar y diagnosticar las fallas. [3] presenta conceptos básicos relacionados con este campo. De acuerdo con [4], la Figura 1 muestra la metodología general empleada para la detección y diagnóstico. El autor describe los modelos de detección y diagnóstico como procesos invasivos y no invasivos. Otro punto de vista se proporciona en [5, 6] que han abordado el problema de la detección y diagnóstico clasificando los métodos en tres categorías diferentes: los modelos cuantitativos que hacen uso de modelos matemáticos, los modelos cualitativos que realizan la detección combinando la teoría de grafos con los modelos matemáticos en alguna parte del sistema, y finalmente, los modelos que hacen uso de los datos históricos para llevar a cabo un sistema de control completo.

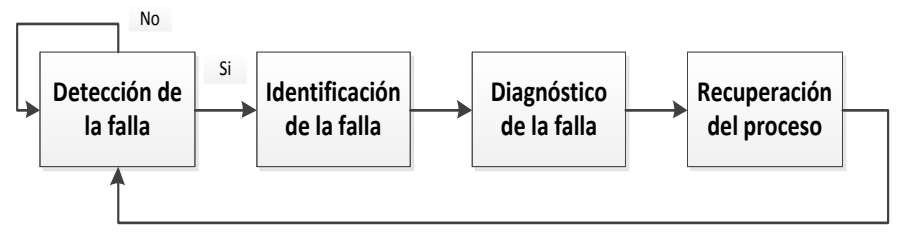

Fig. 1. Sistema de control de procesos (Adaptado de [4]).

Los métodos basados en datos históricos del proceso, son ampliamente utilizados hoy en día en la mayoría de los procesos o sistemas debido a su relativa simplicidad y eficiencia [7] y [8]. En el caso particular de los sistemas eléctricos, las técnicas de computación suave, tales como: la lógica difusa, redes neurales artificiales, razonamiento probabilístico y algoritmos evolutivos se emplean como clasificadores. [9] utiliza la lógica difusa para hacer el proceso de detección de fallas en tiempo real 
en un sistema de inyección de combustible en un motor diesel. [10] propone un sistema de control de nivel de tres tanques con el uso de señales de varios sensores. El objetivo principal de este sistema es detectar señales anormales en los sensores, el uso de lógica difusa es para llevar a cabo la detección de fallas y la fase de diagnóstico se realiza al evaluar la medición de los datos proporcionados. Las distancias euclidianas se utilizan como en $[11,12]$. La primera se aplica en reglas de decisión en la etapa de fotolitografía en el proceso de fabricación de un circuito integrado. El segundo calcula las distancias en un caso de estudio relacionado con los semiconductores a fin de dar el diagnóstico de las fallas. Otro tipo de métrica es la distancia de Mahalanobis, la cual es utilizada por [13] como un sistema de diagnóstico para la viscosidad de la sangre. [14] utiliza estas distancias para analizar los datos obtenidos de la vibración de los rodamientos rígidos de bolas del tipo 62052RS SKF, para identificar rodamientos defectuosos. [15] utiliza como herramienta de diagnóstico metodologías de inteligencia artificial tales como las redes neuronales probabilísticas, utilizadas en la detección de fallas en transformadores. Utilizando los resultados entregados por ensayos realizados sobre el aceite de un transformador, a través del análisis de los gases disueltos, [16] para el caso de un análisis de vibraciones para el diagnóstico de fallas entre máquinas de inducción utiliza una red neuronal probabilística.

\section{Preliminares matemáticos}

Para conocer los preliminares matemáticos correspondientes a Lógica Difusa y las Métricas ver [1,2], puesto que ambas referencias son trabajos previos realizados por los autores.

\subsection{Red neuronal probabilística}

La red neuronal probabilística se compone de dos capas y parte de un vector de datos $p$ expresado de la siguiente manera.

$$
\left\{p_{1}, p_{2}, \ldots, p_{Q},\right\}
$$

Se tiene una matriz $W^{\prime}$ de pesos transpuestos y un vector de tendencia $b$ para una capa cuando:

$$
W^{\prime}=\left[\begin{array}{c}
w_{1}^{T} \\
w_{2}^{T} \\
\vdots \\
w_{s}^{T}
\end{array}\right]=\left[\begin{array}{c}
p_{1}^{T} \\
p_{2}^{T} \\
\vdots \\
p_{Q}^{T}
\end{array}\right], b^{\prime}=\left[\begin{array}{c}
R \\
R \\
\vdots \\
R
\end{array}\right]
$$

La fila de $W^{\prime}$ representa un vector prototipo que se desea reconocer y cada elemento de $b^{\prime}$ es un conjunto igual para el número de elementos en cada vector de entrada $(R)$. Así la salida para la primera capa es: 


$$
a^{1}=W^{\prime} p+b^{\prime}=\left[\begin{array}{c}
p_{1}^{T} p+R \\
p_{2}^{T} p+R \\
\vdots \\
p_{Q}^{T} p+R
\end{array}\right]
$$

La segunda capa es una capa competitiva. Las neuronas en esta capa, se inicializan con las salidas de la capa anterior hacia delante. Que inician el reconocimiento entre los patrones prototipo y el vector de entrada. Estas neuronas compiten con cada una de las otras para determinar un ganador. Después, una sola neurona tendrá una salida distinta a cero. La neurona ganadora indica que categoría está presentando la entrada para la red (cada vector prototipo representa una categoría).

La salida $a^{1}$ de la primera capa es usada para iniciar la segunda capa

$$
a^{2}(0)=a^{1}
$$

La salida de esta segunda capa es actualizada de acuerdo a la siguiente relación de recurrencia.

$$
a^{2}(t+1)=\operatorname{poslin}\left(W^{2} a^{1}(t)\right)
$$

Los pesos de la segunda capa $W^{2}$ se establecen para que los elementos de la diagonal sean $1 \mathrm{y}$ los elementos que se encuentran fuera de la diagonal sean valores negativos [17].

$$
W_{i j}^{2}=\left\{\begin{array}{c}
1, \text { si } i=j \\
-\varepsilon, \text { en otro caso }
\end{array}, \text { cuando } 0<a<\frac{1}{s-1}\right\}
$$

\section{Descripción de la metodología}

En este trabajo se propone una nueva metodología para realizar la detección y diagnóstico de las fallas en un SEP propuesto por la IEEE. La Figura 2 muestra la arquitectura de la propuesta. La metodología consiste en dos pasos. En el primer paso se realiza el proceso de detección, utiliza para ello un sistema de lógica difusa que evalúa las condiciones de funcionamiento del sistema. El segundo paso arroja el diagnóstico final mediante el empleo de una red neuronal probabilística. En el segundo paso se obtiene un patrón de los comportamientos del sistema tanto el modo normal así como del modo anormal de operación entre las tensiones de cada uno de los 24 nodos que forman el sistema eléctrico. Por lo tanto cuando una falla está presente la red neuronal clasifica las distancias euclidianas para dar el diagnóstico correcto e identificar qué línea o líneas presenta una falla ya sea simétrica o asimétrica.

Los pasos de la propuesta se resumen de la siguiente manera:

1. Obtener bases de datos del sistema para el modo de operación normal y para los diferentes tipos de falla que se puedan presentar en él. 
2. Generar un sistema difuso que evalúa cada uno de los nodos del sistema. Se sensibiliza el sistema difuso para entregar un valor de 0.5 cuando el sistema monitoreado se encuentra en modo de falla. En caso de que el sistema se encuentre en modo de operación normal, el sistema difuso entrega un valor distinto de $0.5 \mathrm{y}$ se toma otro conjunto de datos por probar.

Si el sistema difuso encuentra presencia de falla, se procede a calcular las distancias Euclidianas y con el patrón generado por estas distancias se entrena la red neuronal probabilística.

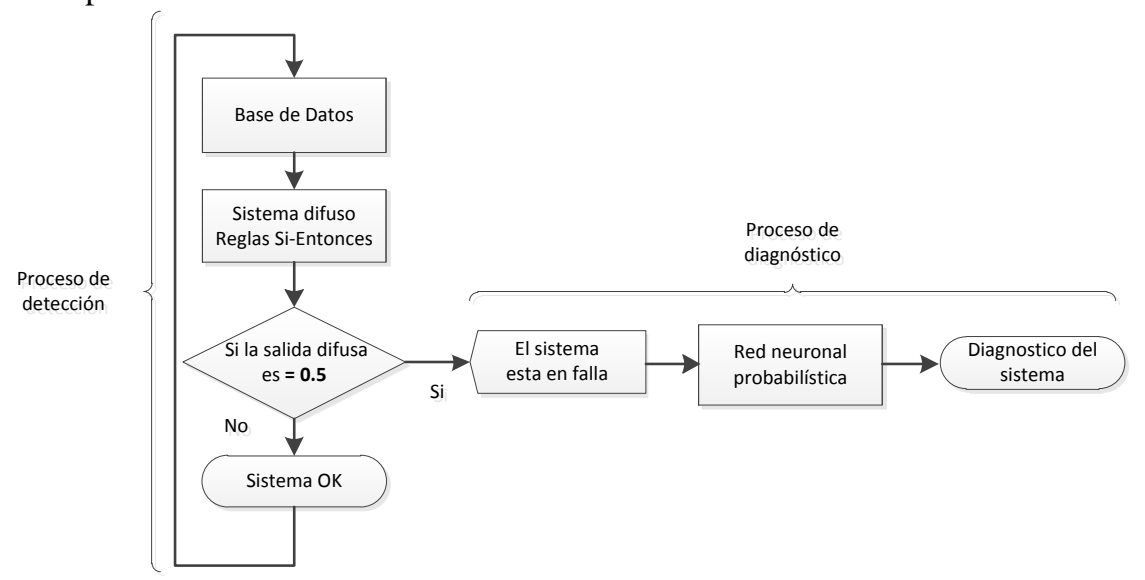

Fig. 2. Metodología general para la detección y diagnóstico de fallas.

3. La red neuronal después de ser entrenada clasifica las distancias y las asigna a un grupo y con ello da un diagnóstico mostrando qué línea se encuentra en modo de falla.

\section{Caso de estudio}

La presente investigación fue encaminada al monitoreo de un SEP con presencia de cambios dinámicos de carga propuesto por la IEEE. El sistema consiste de 24 nodos con sus correspondientes 3 líneas cada uno. Lo cual es un total de 72 variables por monitorear. La Figura 3 muestra dicho sistema eléctrico.

Para el proceso de detección se emplea un sistema difuso. Dicho sistema se entrenó únicamente con bases de datos en modo de operación normal. Esto representa una gran ventaja, ya que no fue necesario entrenar a 24 diferentes sistemas difusos, ni aprender los diferentes modos de falla que se pudieran presentar en el SEP. Para las simulaciones se consideraron dos tipos de fallas. Fallas simétricas y asimétricas, las primeras se producen al juntarse dos líneas entre sí. Las segundas se producen cuando una o más líneas caen a tierra. Las fallas se presentan en la figura 4.

La metodología propuesta se aplicó de la siguiente manera.

El primer paso es el proceso de detección, el cual está compuesto por los bloques mostrados en la Figura 5. 
1. El primer bloque indica que el primer paso es la adquisición y análisis de los datos históricos del sistema eléctrico que se ha descrito anteriormente. El análisis llevo a cabo la evaluación de las amplitudes de las tensiones de las líneas de cada nodo. Se tomaron ventanas de 50 datos con muestras en modo de operación normal y en el modo de falla.

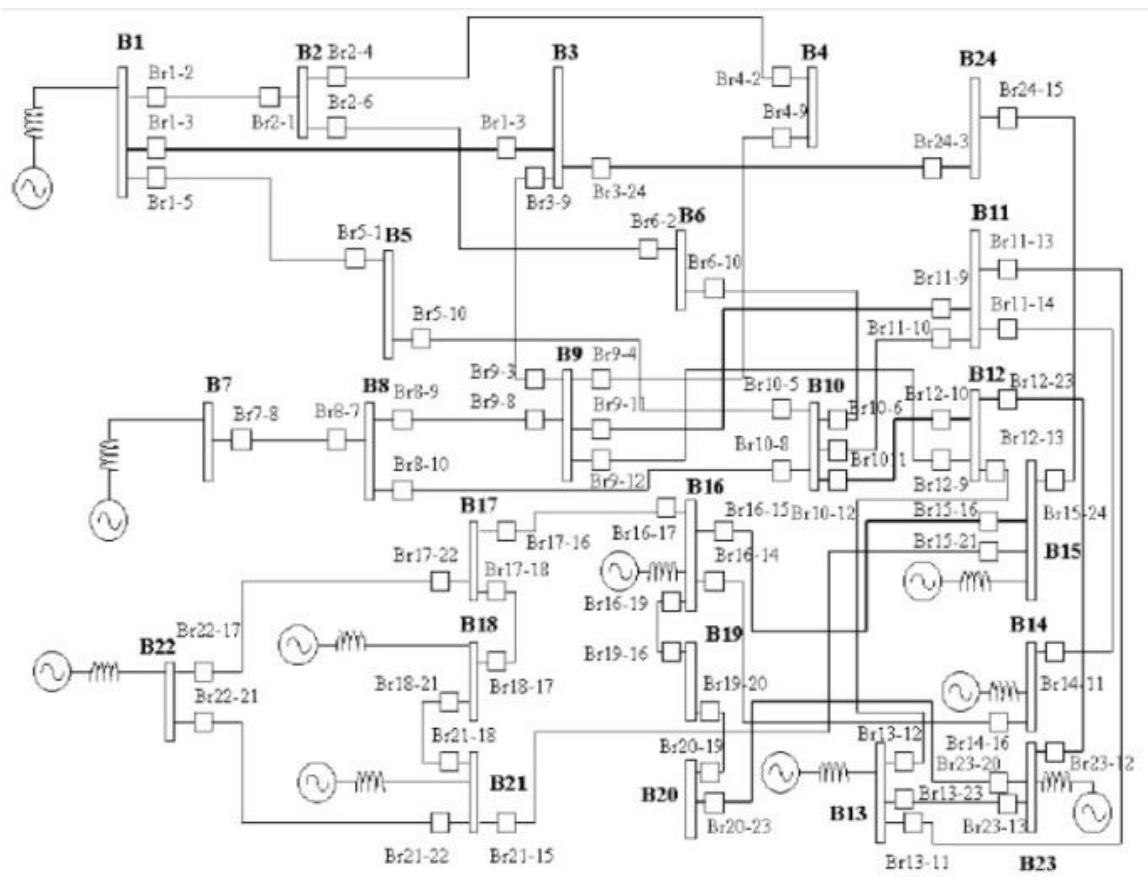

Fig. 3. Diagrama unifilar del sistema propuesto por el IEEE (Adaptado de [18]).
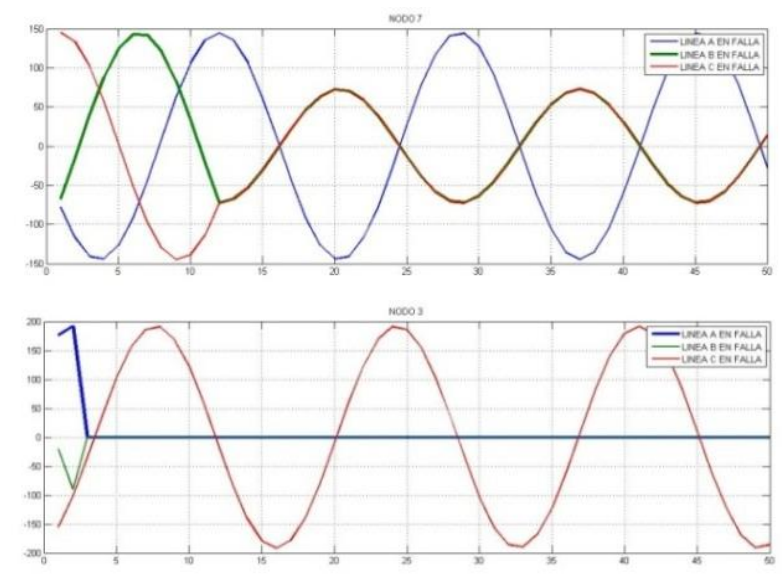

Fig. 4. Falla Simétrica y Asimétrica. 
El siguiente bloque contiene las reglas difusas de los pasos 2 a 6 que se describen a continuación.

En este bloque es necesaria la selección de una función de pertenencia. Se selecciona funciones triangulares debido a su simplicidad.

2. Con las bases de datos tomados en el paso 1, se generaron las reglas difusas que describen el comportamiento del sistema en modo de operación normal. Para lo anterior se generaron un total de 76 reglas difusas que simulan el comportamiento en modo de operación normal del sistema como se muestra en la Tabla 1.

3. Los operadores difusos e implicación difusa seleccionados son los representados en las ecuaciones (2) a (6) de [1, 2]. Lo anterior debido a que dichos operadores e implicaciones pudieron explicar relativamente bien el comportamiento del sistema.

El rango considerado para las amplitudes de las tensiones observadas en cada línea es de -200 a 200 volts.

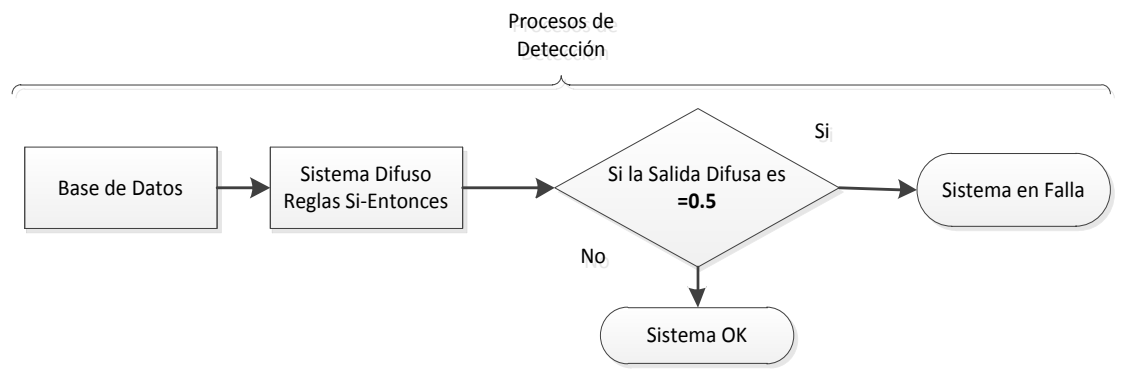

Fig. 5. Metodología del proceso de detección de fallas.

Tabla 1. Reglas difusas.

\begin{tabular}{cccc}
\hline REGLA & \multicolumn{3}{c}{ LINEAS } \\
\hline \hline $\mathrm{N}^{\circ}$ & L1 & L2 & L3 \\
\hline \hline 1 & 150,200 & $-150,200$ & $0,-50$ \\
\hline \hline 2 & 150,200 & $-150,200$ & 0,50 \\
\hline \hline 3 & 50,100 & $-150,200$ & 50,100 \\
\hline \hline$\vdots$ & $\vdots$ & $\vdots$ & $\vdots$ \\
\hline \hline 74 & 50,100 & $-100,150$ & 50,100 \\
\hline \hline 75 & 0,50 & $-100,150$ & 100,150 \\
\hline 76 & $0,-50$ & $-100,150$ & 100,150 \\
\hline
\end{tabular}

Se ha seleccionado el método centroide para llevar a cabo la etapa de defuzzificación descrita en la ecuación (7) de [1] y [2]. Este método es seleccionado debido a que es de los más comúnmente utilizados en el estado del arte.

4. La salida del sistema difuso se sensibiliza para arrojar un valor de 0.5 cuando el sistema se encuentra en un modo de falla. En caso contrario, dará una salida distinta de 0.5 indicando así un modo de operación normal del SEP. 
5. Si se determina que existe una falla se procederá a realizar el segundo paso, si no, se considera que el sistema esté en un modo de operación normal y se procede a analizar la siguiente ventana de datos.

El segundo paso es el proceso de diagnóstico, el cual se lleva a cabo como se describe en las Figuras 6, 7 y 8.

Después de analizar la condición de operación del sistema y una vez detectada la presencia de una falla se procede a encontrar cual es el tipo de falla y en qué elementos se encuentra presente. Tal búsqueda se fundamentó en una primera instancia en la relación presente en las distancias entre las amplitudes de los elementos que se encuentran en modo de falla. A continuación se describen estas relaciones, primero se especifica cómo se lleva a cabo para la presencia de una falla simétrica y posterior mente para una falla asimétrica.

- Fallas simétricas

Para el caso de la falla simétrica la falla no se puede presentar en una sola línea, ya que la falla se presenta cuando se unen dos líneas entre sí. Como un ejemplo se presenta

- Dos líneas en falla simétrica.

Si las distancias entre las líneas L1-L2 es diferente a L2-L3 y L1-L3 y L2-L3 y L1L3 son iguales hay una falla a L1 y L2, y este patrón se repite para los demás casos, como se muestra en la Figura 6.

Por lo tanto, si se cumple alguna de estas condiciones propuestas en esta sección del segundo paso, con las distancias obtenidas por medio del cálculo de las distancias Euclidianas y de Mahalanobis, se determina qué se tiene una falla simétrica presente en él sistema.

- Fallas asimétricas o a tierra

- Una de las líneas con falla a tierra.

Si al comparar las distancias entre L1-L2-L3, las distancias de L2-L3 son valores iguales, entonces la falla está presente en la línea L1. Esto se realiza de manera similar para las demás líneas.

- Dos líneas en falla a tierra.

Si las distancias entre las distancias L1-L2 son 0 y las distancias L2-L3 y L1-L3 son iguales hay una falla a tierra en L1 y L2, como se muestra en la Figura 7.

Por lo tanto, si se cumple alguna de estas condiciones propuestas en esta sección del segundo paso, para las distancias obtenidas por medio del cálculo de las distancias Euclidianas y de Mahalanobis, se determina qué se tiene una falla asimétrica presente en él sistema.

Una de las problemáticas que se tiene al utilizar estas métricas es que con ellas para realizar un correcto diagnostico se tienen que tener todos los elementos de la 
muestra con presencia de falla ya que si estos no presentan este comportamiento, el patrón generado no se cumplirá. Para hacer un diagnóstico más rápido y no depender de una ventana de datos con presencia de falla en todos sus elementos, se utilizó el patrón generado por la distancia Euclidiana entre líneas para caracterizar las fallas en el sistema, y con ello se propuso entrenar una red neuronal probabilística para hacer la clasificación de la falla presente e identificar cual línea tiene la presencia de falla, con el fin de hacer el proceso de detección y diagnóstico más eficiente y evitar la presencia de falsas alarmas. La metodología propuesta para la clasificación utiliza una red neuronal la como se muestra en la Figura 8.

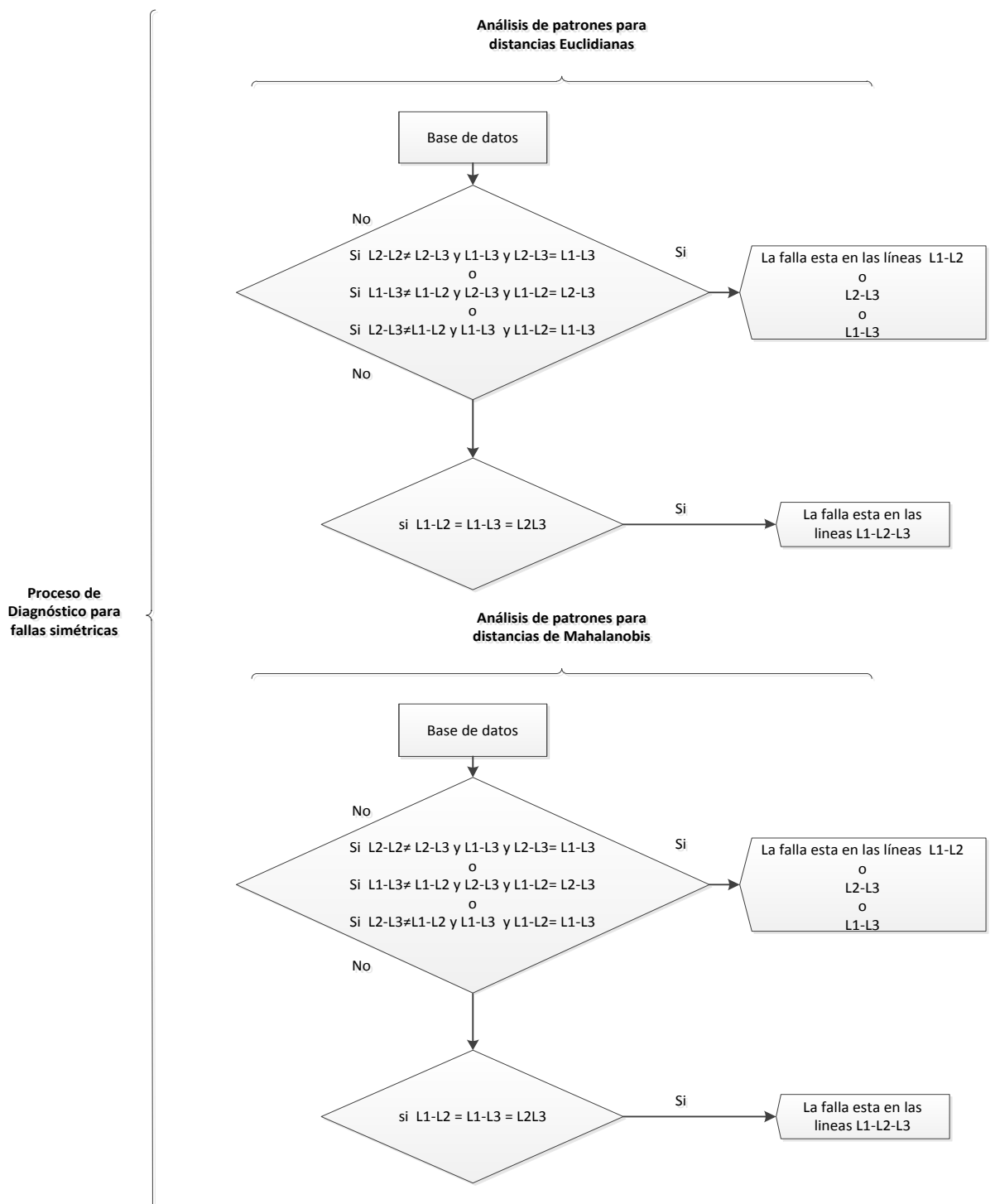

Fig. 6. Metodología para el diagnóstico de fallas Simétricas. 
En la Tabla 2 se valida la metodología descrita. El sistema logra detectar la presencia de fallas en un $100 \%$ de las veces, pero en la etapa de diagnóstico el porcentaje decrece en las distancias debido a que la metodología necesita tener presente en la ventana de 50 datos, todos los datos en modo de falla para hacer un correcto diagnóstico.

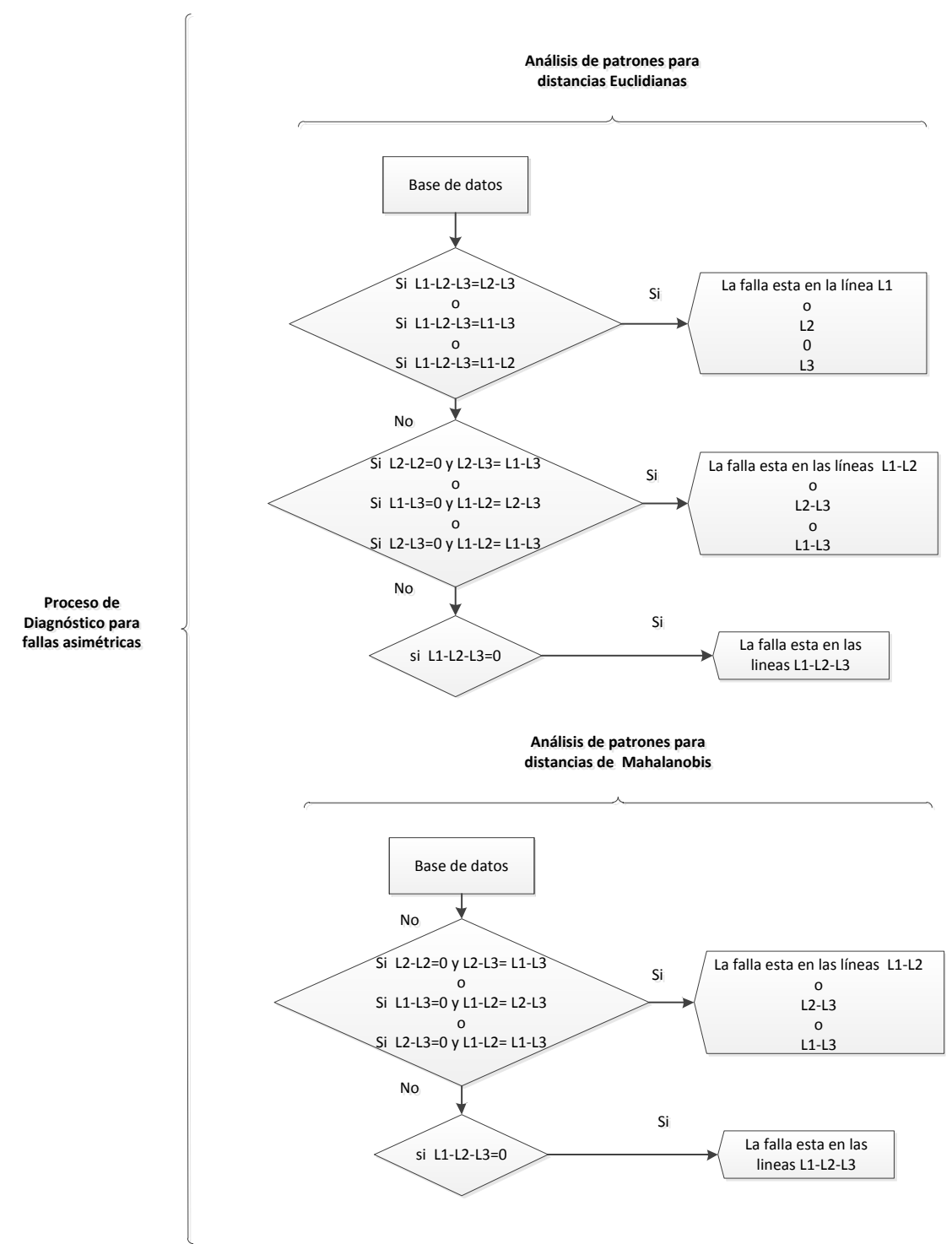

Fig. 7. Metodología para el diagnóstico de fallas Simétricas y Asimétricas.

Como se aprecia en la tabla para evitar esta deficiencia en las métricas, se genera un diagnóstico más preciso del $99 \%$ utilizando una red neuronal probabilística para la clasificación, este porcentaje es alcanzado al utilizar la distancia euclidiana entre 
líneas y con ello se genera un patrón que caracteriza de una mejor manera las fallas y en qué línea están presentes dichas fallas. La red neuronal se entrenó con las distancias con ventanas de 50 datos, como se realizó con el sistema difuso y de igual forma la red neuronal se replicó en los demás nodos del sistema eléctrico y con ello se facilita la detección de fallas múltiples del sistema, la red se validó con 120 datos los cuales presentan los dos tipos de falla en sus líneas así como el modo de operación normal. La red neuronal que se generó cuenta con cuatro entradas para cada una de las distancias euclidianas obtenidas, una capa oculta la cual es entrenada con las distancias que presentan un comportamiento en modo normal de operación y de los casos de falla que se han presentado en el sistema. Una segunda capa con 12 neuronas la cual realiza la clasificación con respecto a la falla presente y una salida que da a conocer el diagnostico requerido, la red neuronal se presenta en la Figura 9.

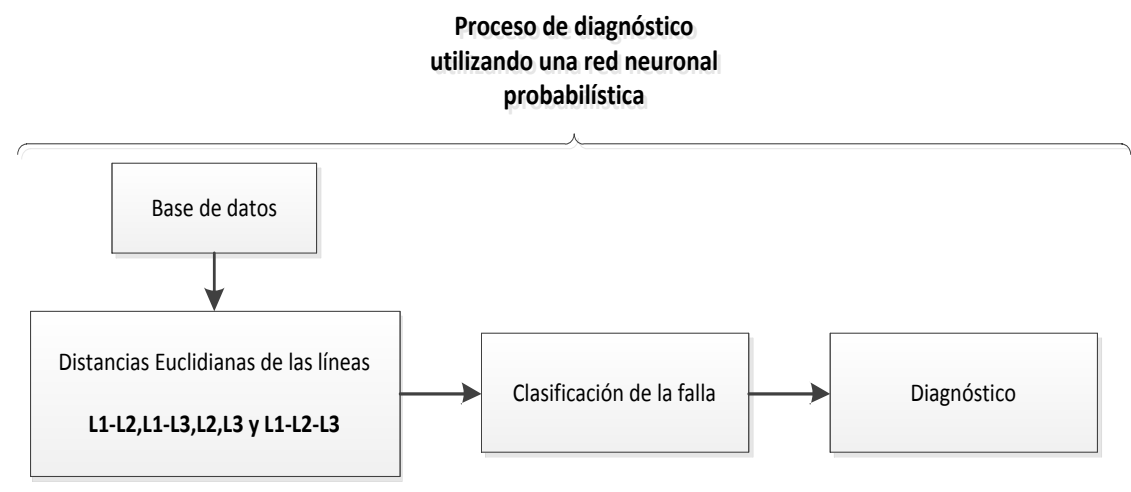

Fig. 8. Metodología para el diagnóstico utilizando una red neuronal probabilística.

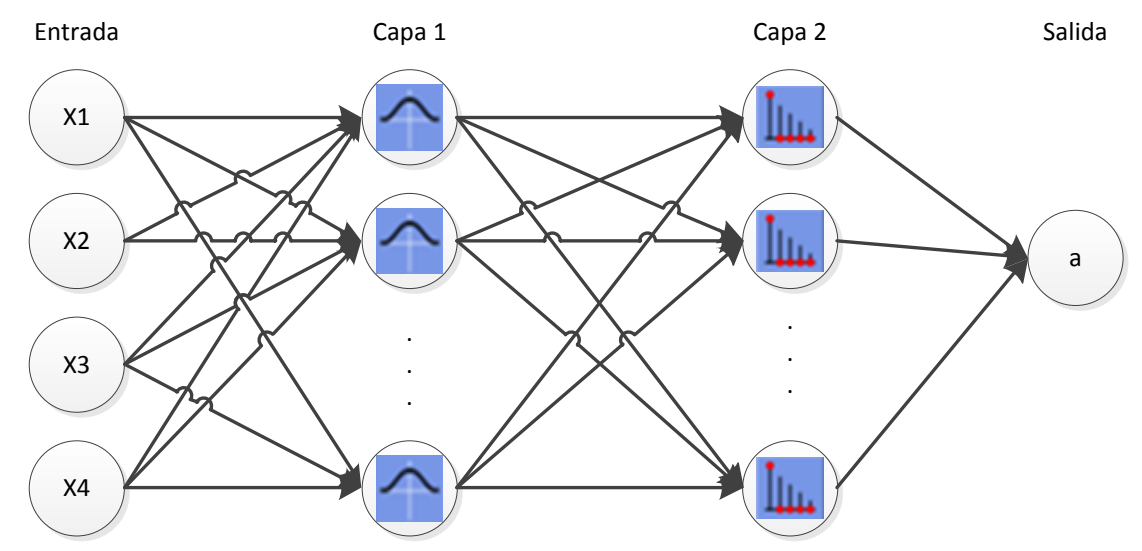

Fig. 9. Red Neuronal Probabilística.

Al determinar el tipo y elementos donde se encuentra la falla en un paso posterior se procederá a hacer la recuperación del sistema para llevarlo nuevamente a su modo normal de operación. 
César Octavio Hernández Morales, Juan Pablo Nieto González, Elías Gabriel Carrum Siller

Tabla 2. Pruebas para validación de la metodología propuesta

\begin{tabular}{ccccc}
\hline $\begin{array}{l}\mathrm{N}^{\circ} \text { Fallas en } \\
\text { Ventanas de } \\
50 \text { Datos }\end{array}$ & $\begin{array}{l}\text { \% en que se } \\
\text { realizó la } \\
\text { Detección }\end{array}$ & $\begin{array}{l}\text { \% para el } \\
\text { Diagnostico } \\
\text { Distancia } \\
\text { Euclidiana }\end{array}$ & $\begin{array}{l}\text { \% para el } \\
\text { Diagnostico } \\
\text { Distancia de } \\
\text { Mahalanobis }\end{array}$ & $\begin{array}{l}\text { \% para el } \\
\text { Diagnostico } \\
\text { Con la Red } \\
\text { Neuronal } \\
\text { Probabilística }\end{array}$ \\
\hline \hline 1 & 100 & 75 & 70 & 99 \\
2 & 100 & 80 & 75 & 99 \\
3 & 100 & 80 & 80 & 99 \\
4 & 100 & 80 & 80 & 99 \\
5 & 100 & 80 & 80 & 99 \\
\hline
\end{tabular}

\section{Conclusiones}

En el presente artículo se ha propuesto un sistema de detección y diagnóstico de fallas basado en datos del histórico del proceso. La propuesta utiliza un sistema de lógica difusa para el proceso de detección y al obtener las distancias Euclidianas genera un patrón para el entrenamiento de una red neuronal probabilística.

Con la red se clasifican los patrones generados por estas distancias para arrojar un diagnóstico final más confiable y con ello disminuir la presencia de falsas alarmas. La metodología propuesta fue validada en un sistema eléctrico de potencia con cambios dinámicos de carga. El SEP monitoreado está compuesto por 24 nodos y es propuesto por la IEEE.

Como se mostró en la tabla 2 se obtiene un diagnóstico más eficiente al utilizar una herramienta inteligente para la clasificación que al utilizar una comparación entre dos tipos de métricas.

\section{Referencias}

1. César Octavio Hernández Morales and Juan Pablo Nieto González: Fault detection and diagnosis of electrical networks using a fuzzy system and euclidian distance. In: 12th Mexican International Conference on Artificial Intelligence, MICAI 2013 Mexico City, Mexico, Proceedings, Part II, pp. 216-224 (2013)

2. César Octavio Hernández Morales and Juan Pablo Nieto González: Detección y Diagnóstico de Fallas en SEP's Combinando Lógica difusa con distancias Euclidianas y de Mahalanobis. En: X Congreso Internacional sobre Innovación y Desarrollo Tecnológico, Cuernavaca, Morelos, México (2014)

3. Julio César Ramírez Valenzuela: Diagnóstico de fallos en sistemas industriales basado en razonamiento borroso y posibilístico. Universidad Politécnica de Valencia, Departamento de Ingeniería de Sistemas y Automática (2007)

4. Ali Ajami, Mahdi Daneshvar: Data driven approach for fault detection and diagnosis of turbine in thermal power plant using Independent Component Analysis (ICA). Electrical Power and Energy Systems, pp. 728-735 (2012) 
5. Venkatasubramanian V., Rengaswamy R., Yin K., Kavuri S.: A review of process fault detection and diagnosis Part I. Computers and Chemical Engineering, 27, pp. 293-311 (2003)

6. Juan Pablo Nieto González, Luis Garza Castañón y Rubén Morales Menéndez: Multiple Fault Diagnosis in Electrical Power Systems with Dynamic Load Changes Using Probabilistic Neural Networks. Computación y Sistemas, 14(1), pp. 17-30 (2010)

7. Cox, Earl: Fuzzy fundamentals. IEEE Spectrum, pp. 58-61 (2002)

8. J.P. Nieto: Multiple Fault Diagnosis in Electrical Power Systems with Dynamic Load Changes Using Soft Computing. In: 11th Mexican International Conference on Artificial Intelligence, MICAI 2012: Advances in Computational Intelligence Proceedings Part 2, pp. 319-330 (2012)

9. Yong He and Lei Feng: Diesel Fuel Injection System Faults Diagnosis Based on Fuzzy Injection Pressure Pattern Recognition. In: Proceedings of the 5th World Congress on Intelligent Control and Automation, Hangzhou, China, pp. 1654-1657 (2004)

10. Seda Postalcio Lu: Signal Processing and Fuzzy Cluster Based Online Fault Diagnosis. pp. 1454-1459 (2009)

11. Ghislain Verdier and Ariane Ferreira: Adaptive Mahalanobis Distance and k-Nearest Neighbor Rule for Fault Detection in Semiconductor Manufacturing. IEEE Transactions on Semiconductor Manufacturing, 24(1), pp. 59-67 (2011)

12. Han Ya-juan, HE Zhen and SONG Guo-fang: Research for Multidimensional Systems Diagnostic Analysis based on Improved Mahalanobis Distance. 2009, IEEE, pp. 213-217.

13. Jinshan Lin and Qian Chen: Fault diagnosis of rolling bearings based on multifractal detrended fluctuation analysis and Mahalanobis distance criterion. Mechanical Systems and Signal Processing, pp. 515-533 (2013)

14. Ming Luo, Ying Zheng and Shujie Liu: Data-based Fault-tolerant Control of the Semiconductor Manufacturing Process based on K-Nearest Neighbor Nonparametric Regression. In: Proceedings of the 10th World Congress on Intelligent Control and Automation, pp. 3008-3012 (2012)

15. Juan Andrés Cadena, Juan Mauricio Cadena y Sandra Milena Pérez: Aplicación de redes neuronales probabilísticas en la detección de fallas incipientes en transformadores. Scientia et technica, vol. XIV, núm. 39, pp. 48-53 (2008)

16. Jeevanand Seshadrinath, Bhim Singh, B. K. Panigrahi: Vibration analysis based interturn fault diagnosis in induction machines. IEEE TRANSACTIONS ON INDUSTRIAL INFORMATICS, VOL. 10, NO. 1, pp. 340-350 (2014)

17. Martin T. Hagan: Neural Network design. Oklahoma State University, Mark Beale MHB, Inc., pp. 1-15 (1996)

18. Juan Pablo Nieto González: Complex Systems Fault Diagnosis Using Soft Computing and Statistical Methods. Instituto Tecnológico y de Estudios Superiores de Monterrey, Campus Monterrey, Escuela de Ingeniería y Tecnologías de Información (2012) 